\title{
UAV Rotorcraft in Compliant Contact: Stability Analysis and Simulation
}

\author{
Paul E. I. Pounds and Aaron M. Dollar
}

\begin{abstract}
A hovering vehicle interacting with objects and surfaces must be robust to contact forces and torques transmitted to the airframe, which produce coupled dynamics distinctly different from those of free flight. These mechanics may be modeled as elastic couplings between the aircraft and the ground, represented by a 6-DOF spring in $\mathbb{R}^{3} \times S O(3)$. We show that Proportional Derivative attitude and position controllers that stabilize a rotorcraft in free flight will also stabilize the aircraft during contact for a range of contact displacements and stiffnesses. Simulation of the coupled aircraft dynamics demonstrates stable and unstable modes of the system.
\end{abstract}

\section{INTRODUCTION}

Unmanned Aerial Vehicles (UAVs) have effectively demonstrated their ability to fly, maneuver and carry out observation tasks in numerous roles. However, they remain passive observers, limiting their interaction with the environment to remote surveillance. The increasing ubiquity of UAVs will be further supported by developing the means for these robots to contact and engage with their surroundings robustly. The ability to manipulate objects while hovering will allow these vehicles to be used for infrastructure maintenance and other similar tasks in locations inaccessible to terrestrial vehicles, such as the tops of power lines and radio masts, rough terrain or water surfaces.

When an aircraft contacts a surface, forces are transmitted to the airframe that may destabilize the vehicle if not properly accounted for. Such perturbations arise both when actively engaging and manipulating objects, as well as during inadvertent collisions. Robustness to these forces during flight is essential for aerial robots performing physical tasks in complex real-world environments; the vehicle must guarantee flight stability throughout all modes of operation.

Aircraft-object interactions have historically been in the form of tethered flight and surface contact in landing, with care taken to avoid pathological coupled modes, such as dynamic rollover [1]. Work on UAV-object interaction has followed these lines of inquiry.

The dynamics of an aircraft tethered to ground are important for landing helicopters on ships. Much work has been done to develop control laws for UAV rotorcraft operating in this regime [2]-[4]. These analyses typically treat tether forces as a constant bias load and present control designs that reject it. However, this is not germane to object interactions where forces are not constant and change dynamically.

This work was supported in part by the Office of Naval Research grant N000141010737.

Paul Pounds is a post-doctoral associate and Aaron Dollar is an assistant professor at the Dept. of Mechanical Engineering, Yale University New Haven CT, 06511, USA. \{firstname. lastname\} @yale.edu

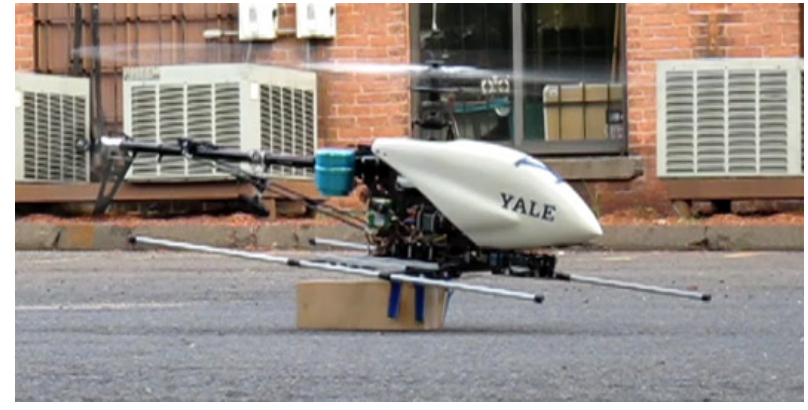

Fig. 1: Yale Aerial Manipulator platform with compliant ventral gripper.

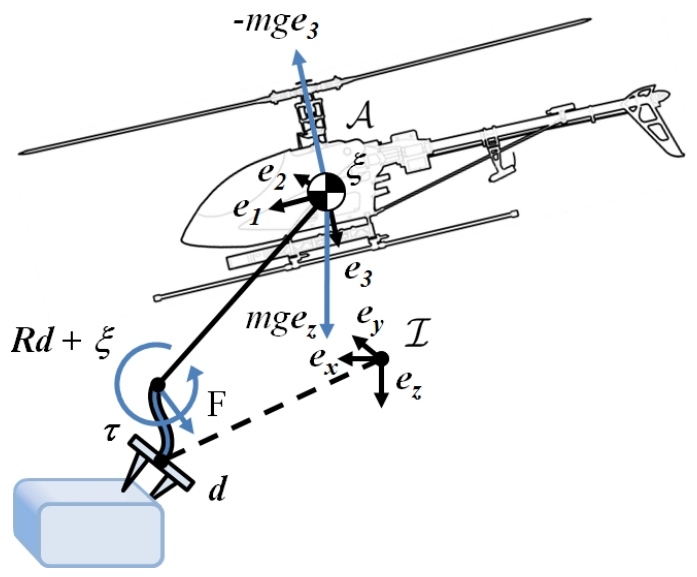

Fig. 2: Aircraft Free Body Diagram.

In addition to ground contact, researchers at Università di Bologna have explored the interaction of a ducted-fan UAV with hard point-contacts flying against sloped and vertical surfaces [5]. Gentili et al employ a state-machine based mode switching controller that dynamically changes its flight control law as the aircraft transitions from one distinct contact configuration to another. This work was extended to a quadrotor model with a similar control strategy [6].

Rather than treat interactions as constant bias or mechanically distinct configurations, our approach is to model contact as serial compliance through an end-effector, probe or other part of the aircraft [7]. This approach is related to the authors' current work utilizing a compliant gripper with both angular and translational stiffness, to grasp and manipulate target objects [8]. This framework also applies to cases such as ground contact with landing gear, pushing objects with landing skids or touching a wall with a rotor shroud.

Commercial off-the-shelf flight stabilizers employing Proportional Derivative (PD) and Proportional Integral Derivative (PID) architectures are increasingly available for UAV rotorcraft. Rather than employ custom designs tailored for object interactions, it is desirable to use these standard controllers to maintain stability during contact. Many com- 
mercial systems are not adjustable mid-flight and therefore the same gains that regulate free flight would ideally continue to stabilize the aircraft in contact. By finding compliance limits that guarantee continued stability and designing the contact mechanism appropriately, the controller's free-air flight performance can be retained without risk of destabilization in contact. Such tuned stiffnesses ensure stability without knowledge about the contact and are effective even during uncertain contact configurations or unexpected collision.

Previously, we examined the dynamic stability of 3-DOF planar helicopters with PID attitude control and rotor flapping mechanics, connected to ground by a link with translational and angular springs [7]. These systems were shown to remain stable within a maximum lateral and angular stiffness ratio. This was demonstrated experimentally with a helicopter equipped with a compliant gripper (see Fig. 1) that hovered stably while gripping a block fixed to ground [8]. The planar case, however, does not consider the more complex interactions arising from cross-coupled motion or contact points not aligned with the rotor axis.

In this paper we extend this structure to 6-DOF, for PD control of both attitude and position, and consider arbitrary end-effector positions. In section II we translate the full planar dynamic model to motion in all axes, simplify this model for control analysis, and present a 6-DOF end-effector compliance model in $\mathbb{R}^{3} \times S O(3)$. In section III we describe attitude and position controllers employing time-scale separation that stabilize the aircraft in free-air. These control laws are used in section IV to derive bounds within which the aircraft will remain stable during contact. Simulations of stable and unstable configurations are presented in section V. Section VI discusses the implications of aircraft stability for aerial manipulation. Section VII concludes the paper.

\section{DyNAMiC MODEL}

The fundamental dynamics of small-scale helicopters are well established [4], [9]-[11]. These models typically include the mechanics of a rotating and translating rigid body, driven by a pair of rotors that produce torques and forces at some offset from the center of mass. We will adapt a similar 6-DOF model and make appropriate simplifications to facilitate control analysis.

\section{A. Free-Air Dynamic Model}

The inertial reference frame is denoted by $\mathcal{I}=\left\{\mathbf{e}_{x}, \mathbf{e}_{y}, \mathbf{e}_{z}\right\}$, where $\mathbf{e}_{z}$ is in the direction of gravity, and $\boldsymbol{\xi}=(x, y, z)$ is the origin of the body fixed frame $\mathcal{A}=\left\{\mathbf{e}_{1}, \mathbf{e}_{2}, \mathbf{e}_{3}\right\}$ where $\mathbf{e}_{1}$ is aligned with the front of the craft (see Fig. 2). The frame $\mathcal{A}$ is related to $\mathcal{I}$ by the rotation matrix $R: \mathcal{A} \rightarrow \mathcal{I}$. Vector $\mathbf{v}$ is the translational velocity of frame $\mathcal{A}$ in $\mathcal{I}$ and $\Omega$ is angular velocity of frame $\mathcal{A}$ expressed in $\mathcal{A}$.

The system dynamic equations are:

$$
\begin{aligned}
\dot{\boldsymbol{\xi}} & =\mathbf{v} \\
m \dot{\mathbf{v}} & =-\boldsymbol{\Omega}_{\times} m \mathbf{v}+m g \mathbf{e}_{z}+R \mathbf{T}_{M}+R \mathbf{T}_{T} \\
\dot{R} & =R \boldsymbol{\Omega}_{\times} \\
\mathrm{J} \dot{\boldsymbol{\Omega}} & =-\boldsymbol{\Omega}_{\times} \mathrm{J} \boldsymbol{\Omega}+\mathbf{T}_{M \times} \mathbf{h}+\mathbf{T}_{T \times} \mathbf{l}+\boldsymbol{\tau}_{M} \mathbf{e}_{3}+\boldsymbol{\tau}_{T} \mathbf{e}_{2}(4)
\end{aligned}
$$

where $m$ and $\mathbf{J}$ are the mass and rotational inertia of the aircraft, $g$ is acceleration due to gravity, $\mathbf{T}_{M}, \boldsymbol{\tau}_{M}, \mathbf{T}_{T}$ and $\boldsymbol{\tau}_{T}$ are the thrust and drag torque vectors produced by the main rotor and tail rotor respectively, and $\mathbf{h}$ and $\mathbf{l}$ are their displacements from the center of mass. Here $\times$ is the skewsymmetric matrix operator.

Thrust vectors are computed as functions of rotor tilt angles. The planar model extended this description to include the effects of blade flapping for a zero-hinge offset 'teetering' rotor. These arise from aerodynamic-centripetal force imbalance due to body translation and rotation in the air (cf. [12]). The definition used in the planar case can be extended to 6-DOF by including the lateral components of flapping and abstracting the distortion of the rotor tip plane to include arbitrary velocities ${ }^{1}$ :

$$
\begin{aligned}
\mathbf{T}_{M}= & -\alpha_{M} u_{4}\left(I+\left[\begin{array}{lll}
-u_{2} & u_{1} & 0
\end{array}\right]_{\times}^{\prime}\right. \\
& \left.-\left(Q_{1} \mathbf{v}_{\times} \mathbf{e}_{3}\right)_{\times}-\left(Q_{2} \boldsymbol{\Omega}\right)_{\times}\right) \mathbf{e}_{3} \\
\mathbf{T}_{T}= & -\alpha_{T} u_{3} \mathbf{e}_{2}
\end{aligned}
$$

where $I$ is the $3 \times 3$ identity matrix, and $\alpha_{M}$ and $\alpha_{T}$ are thrust scaling of the rotors with blade angle of attack. The control inputs $u_{i}$ are the absolute rotor blade pitch angle controls: $u_{1}$ and $u_{2}$ are the lateral and longitudinal rotor cyclic pitch, and $u_{3}$ and $u_{4}$ are the tail rotor and main rotor collective pitch. Matrices $Q_{1}$ and $Q_{2}$ are constant translation and rotation flapping parameters of the rotor, respectively:

$$
\begin{aligned}
& Q_{1}=q_{1}\left(\begin{array}{lll}
\mathbf{e}_{1} & \mathbf{e}_{2} & 0
\end{array}\right) \\
& Q_{2}=q_{2}\left(\begin{array}{lll}
\mathbf{e}_{1} & \mathbf{e}_{2} & 0
\end{array}\right)+\frac{1}{\omega_{0}}\left(\begin{array}{lll}
\mathbf{e} e_{2} & \mathbf{e}_{1} & 0
\end{array}\right)
\end{aligned}
$$

where $q_{1}$ and $q_{2}$ are the same rotor translation and rotation parameters used in the planar model [7] and $\omega_{0}$ is the angular velocity of the rotor.

\section{B. Analytical Model}

The model given above is directly solvable in the planar case, but difficult to analyze in 6-DOF. We apply simplifications to the model that yield an easier system to analyze, but which retains its essential behavior. The complete model is used for the simulations in section $\mathrm{V}$.

Around equilibrium flight conditions the set-point rotor control angles will induce torques and forces that exactly cancel the constant forces on the airframe (e.g. tail-rotor thrust moment balances main rotor drag torque). System control inputs can be regarded as the sum of the equilibrium values, plus a variation around these points: $u_{i}=u_{0 i}+\delta u_{i}$. We can cancel these steady-state contributions and only consider the effect of the variational inputs.

Dimensional analysis applied to the planar case indicates that under closed loop control around equilibrium the influence of flapping mechanics on system stability is small. Mahony et al note that the body forces due to rigid body motion, $-\boldsymbol{\Omega}_{\times} m \mathbf{v}$, and the lateral forces induced by $\delta u_{1}$

\footnotetext{
${ }^{1}$ This generalizes the model given in [11], which represented rotor flapping as a transformation between body velocity and locally observed rotor wind velocity
} 
and $\delta u 2$ are small and can be ignored [9]. These forces are omitted from the analysis, but are included in the simulation. The stabilizing effects of rotor inflow damping and ground effect cannot be assumed and are excluded.

Importantly, these approximations impart a strict hierarchal structure to the dynamics, with no feed-forward mechanics. Consequently, inputs $\delta u_{i}$ act only to induce torques; we combine these system inputs into a single control vector $\boldsymbol{\Gamma}=\left[\begin{array}{lll}\delta u_{2} & \delta u_{1} & \delta u_{3}\end{array}\right]^{\prime}$. The simplified dynamics we will use for analysis are of the standard form:

$$
\begin{aligned}
\dot{\boldsymbol{\xi}} & =\mathbf{v} \\
m \dot{\mathbf{v}} & =m g \mathbf{e}_{z}-T R \mathbf{e}_{3} \\
\dot{R} & =R \boldsymbol{\Omega}_{\times} \\
\mathbf{J} \dot{\boldsymbol{\Omega}} & =-\boldsymbol{\Omega}_{\times} \mathbf{J} \boldsymbol{\Omega}+\boldsymbol{\Gamma}
\end{aligned}
$$

where $T=m g+\alpha_{M} \delta u_{4}$.

\section{Contact Model}

Consider a UAV equipped with an end-effector mounted some displacement $\mathbf{d}$ from its center of mass. When the endeffector comes into contact with an object or surface, the dynamics of the closed-loop system will change. We represent contact and interaction with objects by an elastic multidimensional coupling; this coupling transmits forces from the aircraft to the object and vice versa. While we are particularly interested in the case where the end-effector has a firm grasp and produces stiffness in all axes, these models may also be adapted for unidirectional surface contact constraints.

The planar model approximated a compliant gripper as a combination of lateral, vertical and angular springs of varying stiffness. Similarly, we consider a multi-dimensional spring that can apply both angular and translational forces.

Josip Lončarić posits that there is no natural positive definite metric on the group of body displacements in $S E(3)$ [13], making realization of a truly generalized elastic element for 6-DOF motion intractable. However, the space $\mathbb{R}^{3} \times$ $S O(3)$ is diffeomorphic to $S E(3)$ and such metrics do exist in $S O(3)$ and $\mathbb{R}^{3}$. Thus we consider a pair of springs: one 3-DOF translational spring and one 3-DOF rotational spring that act on the body to produce a force and torque (see Fig. 3).

$$
\begin{aligned}
\mathbf{F}_{\xi} & =-K_{\xi}(R \mathbf{d}-\mathbf{d}+\boldsymbol{\xi}) \\
\boldsymbol{\tau}_{R} & =-K_{R} \log (R)
\end{aligned}
$$

where $K_{\xi}$ and $K_{R}$ are translational and rotational spring stiffness matrices, and $\log (R)$ is the $\mathfrak{s o}(3)$ mapping of the matrix logarithm of $R$ :

$$
\log (R)=\theta \boldsymbol{\omega}
$$

where $\theta$ and $\boldsymbol{\omega}$ are the angle-axis pair of the exponential representation of the body attitude [14]:

$$
\begin{aligned}
\theta & =\arccos \left(\frac{\operatorname{trace}(R)-1}{2}\right) \\
\boldsymbol{\omega} & =\frac{1}{2 \sin (\theta)}\left(\begin{array}{l}
R_{32}-R_{23} \\
R_{13}-R_{31} \\
R_{21}-R_{12}
\end{array}\right)
\end{aligned}
$$

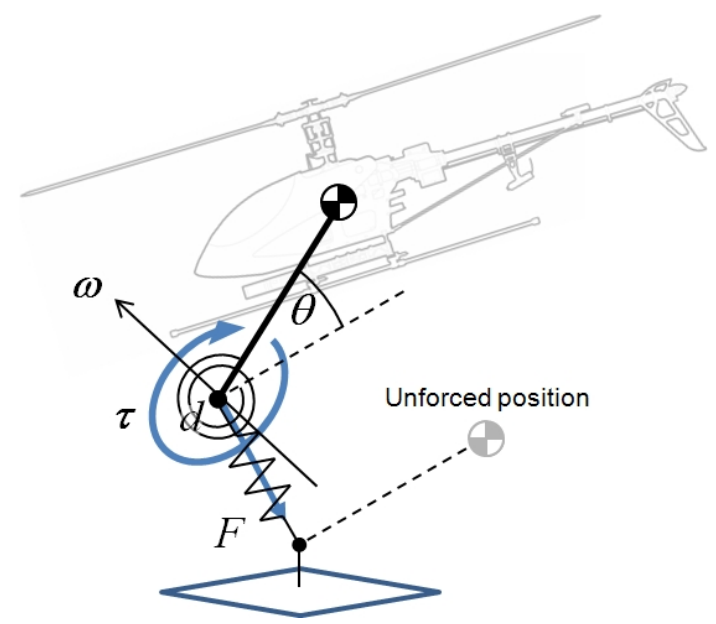

Fig. 3: Contact Spring Configuration.

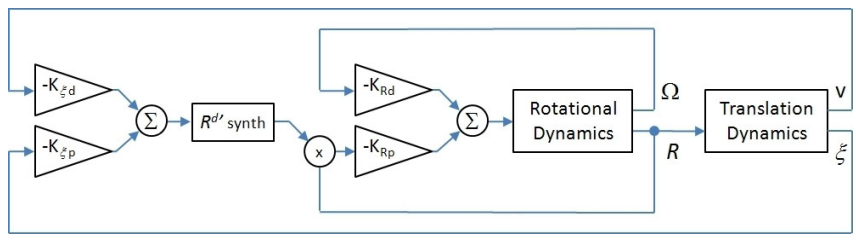

Fig. 4: Flight Control Architecture.

When $\xi=0$ and $R=I$, the end-effector is touching the object with zero force and torque.

As the end-effector compliance is offset from the aircraft center of mass by $\mathbf{d}$, the forces and torques are coupled. The translational spring force depends on displacement of the end effector, a function of $R$. The force induces a moment about the center of mass:

$$
\boldsymbol{\tau}_{\xi}=K_{\xi}(R \mathbf{d}-\mathbf{d}+\boldsymbol{\xi})_{\times} \mathbf{d}
$$

Similarly, the rotational spring torque induces forces on the body:

$$
\mathbf{F}_{R}=K_{R} \mathbf{d}_{\times} \log (R)
$$

\section{Closed-Loop Free-Air Stability}

Aircraft flight controllers are designed to stabilize the vehicle in free-air. Attitude stability is most crucial as rotorcraft require near-level pose to remain in the air, but translational stability is also important. A common architecture uses a cascaded loop structure in which attitude is stabilized first, and then position. We consider a flight controller employing PD control to stabilize each loop (see Fig. 4).

We make the key assumption that the two sets of dynamics are time-scale separated. Small-scale helicopters typically have pitch and roll dynamics that are an order of magnitude faster than their translational dynamics [7], [10]. For systems with high attitude control authority and low position-control bandwidth, this is a reasonable expectation. In general, however, the interaction between pitch and translation oscillatory modes is non-trivial and to be avoided.

We apply the analysis by Bullo and Murray for stabilizing a rigid body on $S O(3)$ and $\mathbb{R}^{3}$ with PD control [14]. They prove stability for second-order systems of this type by 


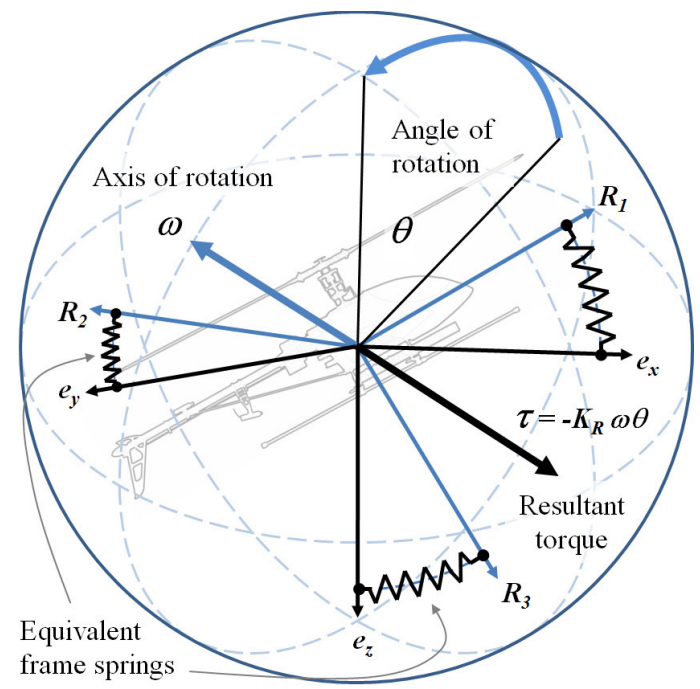

Fig. 5: $S O(3)$ Geodesic Displacement Torque.

constructing Lyapunov energy functions for the angular and translational motion and showing that the derivatives of these functions are negative definite. The attitude dynamics of (12) are exponentially stabilized at $R=I$ by a controller of the form:

$$
\boldsymbol{\Gamma}=-f(R, \boldsymbol{\Omega})-K_{R p} \log (R)-K_{R d} \boldsymbol{\omega}
$$

where $f \in \mathfrak{s o}(3)$ is the "internal drift" arising from system coriolis forces $[\mathrm{J} \boldsymbol{\Omega}, \boldsymbol{\Omega}]$ and $K_{R p}$ and $K_{R d}$ are symmetric positive-definite gain matrices, provided:

$$
\lambda_{\min }\left(K_{R p}\right)>\frac{\|\boldsymbol{\Omega}(0)\|^{2}}{\pi^{2}-\|R(0)\|_{S O(3)}^{2}}
$$

where $\lambda_{\min }$ is the smallest eigenvalue of $K_{R p}, \boldsymbol{\Omega}(0)$ and $R(0)$ are the initial velocity conditions, and $\|R\|_{S O(3)}=$ $\langle\log (R), \log (R)\rangle^{\frac{1}{2}}$ is the ad-invariant metric on $S O(3)$ that gives the arc distance between $R$ and $I$.

In the case where the proportional gain is $K_{R p}=k_{R p} I$, the properties of the skew-symmetric operator and passivity of the coriolis forces allows the controller to be reduced to pure PD form, and the stable system posed in the same structure as (12):

$$
\mathbf{J} \dot{\boldsymbol{\Omega}}=-\boldsymbol{\Omega}_{\times} \mathrm{J} \boldsymbol{\Omega}-k_{R p} \log (R)-K_{R d} \boldsymbol{\Omega}
$$

The $K_{R p} \log (R)$ term is a torque proportional to the minimum geodesic distance between the inertial basis frame and the rotated body coordinate frame - conceptualized as the torque generated by such reference frames with springs attached at each basis vector (see Fig. 5).

If time-scale separation between the attitude and translation dynamics is asserted, translational mechanics can be treated independently from rotation. Position control is implemented by a trajectory of $R$ and $\delta u_{4}$ that produces desired horizontal and vertical forces. It is straightforward to see that a PD control function will stabilize position:

$$
m \dot{\mathbf{v}}=-K_{\xi p} \boldsymbol{\xi}-K_{\xi d} \mathbf{v}
$$

where $K_{\xi p}$ and $K_{\xi d}$ are the positive-definite gains of normalized control function:

$$
\mathbf{U}_{\xi}=-\frac{1}{m g}\left(K_{\xi p} \boldsymbol{\xi}+K_{\xi d} \mathbf{v}\right)
$$

The required $R$ trajectory to generate this control can be determined by constructing the matrix $R^{d}$ :

$$
R^{d}=\left(\begin{array}{lll}
\mathbf{R}_{1}^{d} & \mathbf{R}_{2}^{d} & \mathbf{R}_{3}^{d}
\end{array}\right)
$$

where:

$$
\mathbf{R}_{3}^{d}=\left(\begin{array}{c}
-\mathbf{e}_{y} \cdot \mathbf{U}_{\xi} \\
\mathbf{e}_{x} \cdot \mathbf{U}_{\xi} \\
\left(1-\mathbf{U}_{\xi}^{\prime} P \mathbf{U}_{\xi}\right)^{\frac{1}{2}}
\end{array}\right)
$$

using orthogonal projection matrix $P=\left(\begin{array}{lll}\mathbf{e}_{1} & \mathbf{e}_{2} & 0\end{array}\right)$, such that $\left\|\mathbf{R}_{3}^{d}\right\|=1$. Choose $\mathbf{R}_{1}^{d}$ such that $\left\|\mathbf{R}_{1}^{d}\right\|=1$ and $\mathbf{R}_{1}^{d} \cdot \mathbf{e}_{y}=0$ to set zero yaw, and $\mathbf{R}_{2}^{d}=\mathbf{R}_{3 \times}^{d} \mathbf{R}_{1}^{d}$ such that the $S O(3)$ group structure of $R^{d}$ is maintained. This structure is resolvable provided $\left\|\mathbf{U}_{\xi} P \mathbf{U}_{\xi}^{\prime}\right\|<1$.

Correspondingly:

$$
\delta u_{4}=\alpha_{M}^{-1} m g\left(\mathbf{e}_{z} \cdot \mathbf{U}_{\xi}-\mathbf{e}_{z}+R_{33}^{d}\right)
$$

applies the required $\mathbf{e}_{z}$ force component.

\section{Stability During Contact}

When the end-effector engages with an object the forces applied by the compliant linkage are added to the dynamic model. It is necessary to find the bounds on contact stiffness that maintain stability in coupled flight. In this section we show that the addition of compliance within specified bounds does not destabilize the vehicle.

The velocity dynamics with elastic forces are:

$$
\begin{aligned}
m \dot{\mathbf{v}}= & -K_{\xi p} \boldsymbol{\xi}-K_{\xi d} \mathbf{v}-K_{\xi}(R \mathbf{d}-\mathbf{d}+\boldsymbol{\xi}) \\
& +K_{R} \mathbf{d}_{\times} \log (R) \\
\mathbf{J} \dot{\boldsymbol{\Omega}}= & -\boldsymbol{\Omega}_{\times} \mathbf{J} \boldsymbol{\Omega}-k_{R p} \log (R)-K_{R d} \boldsymbol{\Omega} \\
& -K_{R} \log (R)-K_{\xi}(R \mathbf{d})_{\times} \mathbf{d}+K_{\xi} \mathbf{d}_{\times} \boldsymbol{\xi}
\end{aligned}
$$

We will analyze the dynamics under contact mechanics as if they behave in a strictly time-scale separated fashion. High-gain attitude control designs, where the action of the control inputs can dominate any cross-coupled forces, make time-scale separation a feasible assumption. In such conditions, further simplifications can be made: rotational contributions in the lateral dynamics are ignored and translational contributions in rotational dynamics are treated as constant.

\section{A. Rotation}

Rotational stability in these conditions requires that (21) remains satisfied for the system without translational disturbance forces and that constant disturbance induced by translational spring force is rejected.

The proportional rotation stiffness and control action are posed in the form of the logarithmic angle-axis displacement of the system. However, the moment induced by the lateral spring force by end-effector rotation is not represented in this way. The $(R \mathbf{d})_{\times} \mathbf{d}$ force-lever structure does not support a moment in the direction of $\mathbf{d}$, and so cannot be exactly 


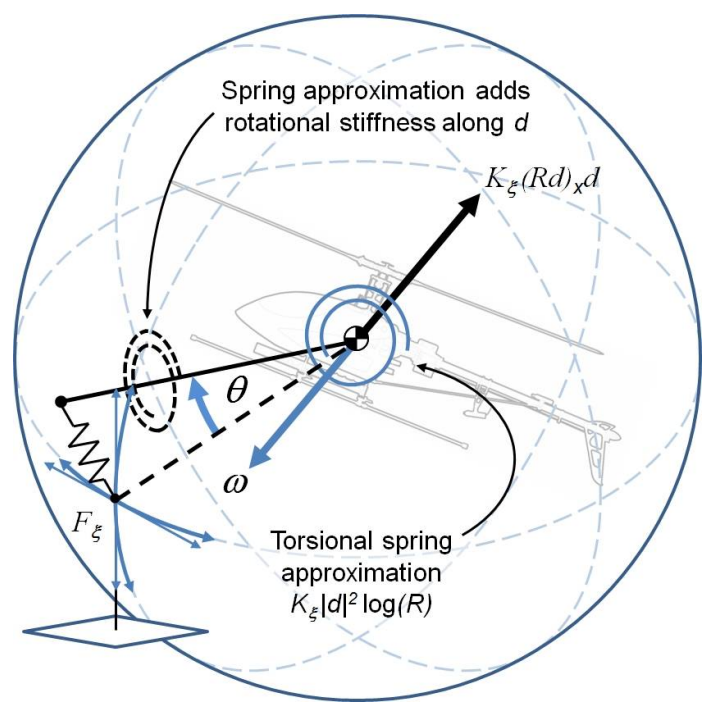

Fig. 6: Translational Spring Rotational Approximation.

expressed as a logarithmic gain. That said, free rotational motion in the d direction is known to be stable without the contributions of spring forces (ie. in free air). We consider, therefore, a more stringent scenario in which rotation in $\mathbf{d}$ is augmented with an additional torsional spring to produce a combined rotational stiffness in the form $K_{\xi}\|\mathbf{d}\|^{2} \log (R)$ (see Fig. 6). The positive-definite gain matrices of the proportional torques can then be directly summed.

It is easy to verify that the eigenvalues of the combined gain must be larger than or equal to those of $k_{R p} I$ alone. Therefore, the addition of proportional elastic rotational forces will not violate (21) and destabilize the aircraft at $I$. However, rotational spring forces work to return the rotation of the body to $I$; to reach $R^{d}$ to apply the translational control action, the proportional angular control will be resisted by the passive rotational elasticity. The proportional control term must dominate, such that eigenvalues of the total effective proportional stiffness matrix $K_{R_{\Sigma}}=k_{R p} I-K_{R}-K_{\xi}\|\mathbf{d}\|^{2}$ also satisfies (21) for some range of $\|R(0)\|$ and $\|\boldsymbol{\Omega}(0)\|$.

Given the constant disturbance moment induced by the translational spring force, the system will not reach equilibrium around $R=R^{d}$. The equilibrium point for this system will instead occur where the combined proportional torques balance the bias torque:

$$
0=-K_{R_{\Sigma}} \log (R)-K_{\xi} \mathbf{d}_{\times} \boldsymbol{\xi}
$$

The steady-state orientation of the system can be directly computed through the rotation matrix exponential map:

$$
R_{\mathrm{B}}=\exp \left\{-K_{R_{\Sigma}}^{-1} K_{\xi} \mathbf{d}_{\times} \boldsymbol{\xi}\right\}
$$

where the mapping $\exp : \mathfrak{s o}(3) \rightarrow S O(3)$ is:

$$
R=I+\sin (\theta) \boldsymbol{\omega}_{\times}+(1-\cos (\theta)) \boldsymbol{\omega}_{\times}^{2}
$$

Within the bound $|\theta|<\pi$, the elastic rotation function is convex - given an equilibrium within this limit, the derivative of the associated energy function will be negative definite. Consequently, the biased system will remain bounded around the equilibrium.

\section{B. Translation}

The translational velocity dynamics with added compliance given in (28) can be rewritten to group constant, proportional and rotation force components. As with the rotational case, force from angular stiffness coupling needs to be recast as a force in the inertial frame. The exponential rotation structure is approximated by $\mathbf{d}_{\times} \theta \boldsymbol{\omega}=\left.\|\mathbf{d}\|\right|^{-1}(\mathbf{d}-R \mathbf{d})$, such that:

$$
\begin{aligned}
m \dot{\mathbf{v}}= & \left(m g \mathbf{e}_{z}+K_{\xi} \mathbf{d}+K_{R}\|\mathbf{d}\|^{-1} \mathbf{d}\right)-K_{\xi} \boldsymbol{\xi} \\
& -R\left(T \mathbf{e}_{3}+K_{\xi} \mathbf{d}+K_{R}\|\mathbf{d}\|^{-1} \mathbf{d}\right)
\end{aligned}
$$

The contributions of the combined translational compliance, $K_{\xi_{\Sigma}}=K_{\xi}+K_{R}\|\mathbf{d}\|^{-1}$, are applied equally to the gravity force and the rotor thrust force. This has the effect of a positive scaling and rotation applied to the two forces; the system remains stable if the off-axis contributions are less than the effective control action:

$$
\frac{\left\|\mathbf{e}_{z \times} K_{\xi_{\Sigma}} \mathbf{d}\right\|}{\mathbf{e}_{z} \cdot\left(m g \mathbf{e}_{z}+K_{\xi_{\Sigma}} \mathbf{d}\right)}<\frac{1}{\sqrt{2}}
$$

that is, where the angle between the desired control action and the applied control action is less than $45^{\circ}$.

\section{Coupled Stability}

From IV-A and IV-B, each second-order subsystem of the dynamics remains locally stable under added stiffness. However, the offset bias in rotation due to $\boldsymbol{\xi}$ couples into translational dynamics by producing non-zero lateral forces.

When aircraft orientation is approximately $I$ and the bias in rotation is small, the exponential map can be approximated by its first order elements $R=I+(\theta \omega)_{\times}$. The bias orientation becomes:

$$
R_{\mathrm{B}}=I+\left(-K_{R_{\Sigma}}^{-1} K_{\xi} \mathbf{d}_{\times} \boldsymbol{\xi}\right)_{\times}
$$

The translational velocity dynamics of (33) can then be written independent of rotation. Canceling constant terms, and combining $m g \mathbf{e}_{z}$ and $m g R \mathbf{e}_{3}$ to implement position control:

$$
\begin{aligned}
m \dot{\mathbf{v}}= & -K_{\xi p} \boldsymbol{\xi}-K_{\xi d} \mathbf{v}-K_{\xi} \boldsymbol{\xi} \\
& -\left(K_{R_{\Sigma}}^{-1} K_{\xi} \mathbf{d}_{\times} \boldsymbol{\xi}\right)_{\times}\left(m g \mathbf{e}_{3}+K_{\xi_{\Sigma}} \mathbf{d}\right)
\end{aligned}
$$

Straightforward manipulation puts this in the form of a proportional system:

$$
\begin{aligned}
m \dot{\mathbf{v}}= & -\left[K_{\xi p}+K_{\xi}-\left(m g \mathbf{e}_{3}+K_{\xi_{\Sigma}} \mathbf{d}\right)_{\times}\left(K_{R_{\Sigma}}^{-1} K_{\xi} \mathbf{d}_{\times}\right)\right] \boldsymbol{\xi} \\
& -K_{\xi d} \mathbf{v}
\end{aligned}
$$

This system will be stable around equilibrium if the eigenvalues of the proportional coefficient are positive. For isotropic stiffness and control gains in the form $K=k I$, these are:

$$
\boldsymbol{\lambda}=\left(\begin{array}{c}
\left(k_{R_{\Sigma}}-m g d_{3}-k_{\xi_{\Sigma}} \mathbf{d}^{\prime} \mathbf{d}\right)\left(k_{\xi p}+k_{\xi}\right) / k_{R_{\Sigma}} \\
\left(k_{R_{\Sigma}}-m g d_{3}-k_{\xi_{\Sigma}} \mathbf{d}^{\prime} \mathbf{d}\right)\left(k_{\xi p}+k_{\xi}\right) / k_{R_{\Sigma}} \\
k_{\xi}
\end{array}\right)
$$

For expected small lateral stiffness and end-effector displacements, the stability condition is approximately $k_{R_{\Sigma}}>m g d_{3}$. 
TABLE I: Standard Yale Aerial Manipulator Parameters.

\begin{tabular}{|c|c|c|c|c|c|}
\hline \multicolumn{6}{|c|}{ Aircraft Parameters } \\
\hline$g$ & 9.81 & $\mathrm{~ms}^{-2}$ & $\mathrm{~J}_{x x}$ & 0.08 & $\mathrm{kgm}^{2}$ \\
\hline$m$ & 3.3 & $\mathrm{~kg}$ & $\mathrm{~J}_{y y}$ & 0.19 & $\mathrm{kgm}^{2}$ \\
\hline $\mathbf{h}$ & {$\left[\begin{array}{lll}0 & 0 & -0.2\end{array}\right]^{\prime}$} & $\mathrm{m}$ & $\mathrm{J}_{z z}$ & 0.19 & $\mathrm{kgm}^{2}$ \\
\hline$q_{1}$ & 0.0039 & & $w_{0}$ & 96 & $\operatorname{rads}^{-1}$ \\
\hline$q_{2}$ & 0.0266 & & & & \\
\hline \multicolumn{6}{|c|}{ Control Parameters } \\
\hline$k_{R p}$ & 1.80 & & $k_{\xi p}$ & 2 & \\
\hline$k_{R d}$ & 1.57 & & $k_{\xi d}$ & 3 & \\
\hline \multicolumn{6}{|c|}{ Standard Gripper Position and Approximate Isotropic Stiffnesses } \\
\hline d & {$\left[\begin{array}{lll}0 & 0 & 0.2\end{array}\right]^{\prime}$} & $\mathrm{m}$ & & & \\
\hline$k_{R}$ & 2.85 & $\mathrm{Nm} / \mathrm{rad}$ & $k_{\xi}$ & 26.6 & $\mathrm{~N} / \mathrm{m}$ \\
\hline
\end{tabular}

\section{Simulation}

Stability bounds (38) and (34) were tested by simulating a variety of end-effector stiffness and position configurations. Base aircraft, control and stiffness parameters used are those of the Yale Aerial Manipulator and its gripper (see Table I). Each simulation starts with $R(0)=I, \boldsymbol{\Omega}(0)=0, \boldsymbol{\xi}=$ $\left[\begin{array}{lll}0 & 0 & -0.5\end{array}\right]^{\prime} \mathrm{m}$ and initial velocity $\mathbf{v}=\left[\begin{array}{lll}0.5 & 0 & 0\end{array}\right]^{\prime} \mathrm{ms}^{-1}$.

We present six archtypical simulations; the standard configuration and five gripper and stiffness variations thereof:

1) The standard configuration. ${ }^{2}$

2) Standard placement, no rotational gripper stiffness.

3) Standard stiffness, gripper $0.2 \mathrm{~m}$ above the CoG.

4) Standard stiffness, gripper $1 \mathrm{~m}$ below the CoG.

5) Standard stiffness, gripper $1 \mathrm{~m}$ ahead of the CoG.

6) Standard stiffness, gripper $1 \mathrm{~m}$ right of the CoG.

Time evolution plots of simulated aircraft position and rollpitch-yaw angles are given in figures 8-13; the ground end of the contact spring is fixed.

As expected from previous experiments with the Yale Aerial Manipulator, simulation 1 shows the aircraft is stable in gripping contact. However, simulation 2 indicates that the same system with no rotation stiffness)will slowly topple, crashing into the ground at $t=5.88 \mathrm{~s}$; this is homologous to dynamic rollover behavior. In contrast, when the gripper is placed at the same distance above the center of gravity, the system oscillates unstably (terminating at $t=4.25 \mathrm{~s}$ ). As the contact point is set far from the CoG system stability diminishes: in simulation 4, rotational stiffness is insufficient to stabilize the aircraft, crashing at $t=3.61 \mathrm{~s}$. Notably, simulations 5 and 6 are not pathological. In simulation 5, the aircraft is excited along the line of the contact probe, producing no cross-coupled disturbance except for small coupling due to flapping, and the system oscillates with stable decay. Simulation 6 shows the aircraft pivot and yaw about its contact point; as the disturbance trajectory is orthogonal to gravity the otherwise unstable motion is arrested by yaw control.

\section{DISCUSSION}

Section IV provides insight into the operation and design of aerial manipulators. Here we discuss how aircraft stability considerations can be utilized in the design of aircraft-endeffector systems and highlight assumptions and limitations of the analysis.

\footnotetext{
${ }^{2}$ Gripper placement and stiffness parameters as per Table I
}

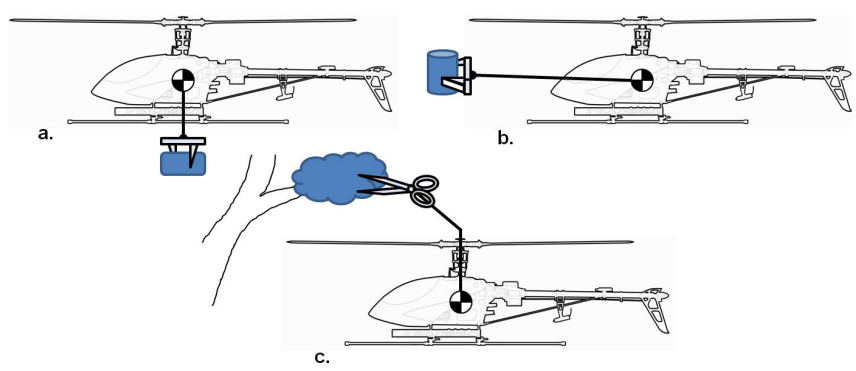

Fig. 7: Possible Contact Configurations: a. $d_{3}>0$, b. $d_{3} \approx 0$, c. $d_{3}<0$.

\section{A. Assumptions, Implications and Limitations}

While the dynamic model given in section II and used in the simulations is comprehensive, the analysis is by no means a complete exploration of the problem. It considers only the most common flight condition of level flight, and many assumptions are made:

- Time-scale separation of dynamical subsystems

- Approximately level flight

- High-gain attitude control, low-gain position control

- Pure rotor torques (zero lateral rotor force contribution)

- Spring force contact approximations.

- No environment compliance, slipping or chattering

The key observation is that aggressive proportional attitude gain is paramount for highly robust stable contact. Rotational end-effector compliance augments the proportional attitude control without influencing free-air performance and so this stiffness should be made large. However, inadvertent contacts are likely to be essentially point contacts, and so a conservative design will rely only on flight control gain.

We identify three major classes of aerial manipulator by the vertical offset of the end-effector $d_{3}$ : contact point substantially below the CoG, substantially above the CoG (e.g. connected through the rotor mast or through the body of a quadrotor), or approximately level with the CoG (see Fig. 7). Given an aircraft and flight-controller with fixed parameters, $d_{3}$ should be minimized so as to reduce the magnitude of torques being transmitted to the attitude dynamics. Free-air stability of laden helicopters with dynamics loads also benefits from small displacements from the CoG [8]. However, this understanding assumes the rotational dynamics of the aircraft in contact reach equilibrium much faster than translational dynamics. Although the planar analysis also showed improved stability performance with shorter endeffector link lengths [7], a resulting increase in coupledsystem natural frequency could be deleterious to stability of non-timescale separated systems.

Notably, equation (38) implies that the system should be inherently stable for some negative values of $d_{3}$; however, (34) permits only a small range of these. The full simulation shows that coupling effects that are benign and ignored in the positive configuration are pathological when the contact point is above the center of mass. In particular, horizontal rotor forces, position control and pitch-translation coupling in the spring destabilize the system, and higher attitude gain serves to exacerbate the effect. Given the rotor-over-body construction of most helicopters, $d_{3}$ is likely to be positive 
or only slightly negative, as an end-effector cannot easily penetrate the disc of the rotor.

Lateral offset of the end-effector tends to induce slow yaw divergence, but as yaw control can be very high-gain in helicopters ${ }^{3}$ lateral offset is not considered pathological.

Further work is needed to reduce the number of assumptions made about aircraft configuration and flight behavior. In particular, understanding of coupled modes that arise from rotation-translation interactions is needed for ensuring complete system stability and to highlight stable above-CoG contact configurations.

\section{B. Object and Surface Manipulation}

A natural extension of the $\mathbb{R}^{3}$ translational compliance model is that forces imparted to an object are proportional to the commanded displacement of the aircraft from $\xi=0$. End-effector position $\mathbf{d}$ is the endpoint of a six-joint manipulator chain - by commanding small translations about the contact point, a flying vehicle can apply translational forces to an object in the environment.

In the case of single-sided contact conditions, such as a probe being pressed against a surface, this model remains valid provided a positive normal contact force is applied. In addition to proper grasping, the ability to apply unidirectional forces through surface contact can be used in applications such sliding or rolling objects too heavy for a small helicopter to lift directly, pressing buttons in human environments, and object retrieval with non-actuated manipulators such as hooks.

Pressing point-contacts, such as in accidental collision, cannot easily support a pure moment at the contact, requiring the flight stabilizer to provide sufficient rotational stiffness. The stability conditions of (21) and (34) do not depend upon any minimum value of $K_{\xi}$. Thus, transition from stable frictional contact to sliding contact or non-contact conditions will not compromise flight stability. The reverse is not necessarily true, as fast contact or impact velocities may violate the dynamics time-scale separation requirement. Contact issues such as stiction, unstable chattering and environment compliance are beyond the scope of this work, but may pose further challenges in ensuring the stability of aircraft.

\section{CONCLUSION}

This paper is a preliminary effort at understanding the problem of a PD-stabilized helicopter manipulating objects, focusing on the most important configuration of compliant contact at low velocities in level hover. This work expanded

\footnotetext{
${ }^{3}$ Quadrotors, which have limited yaw control authority, are expected to be especially susceptible to lateral offset instability, however.
}

the previously presented planar aircraft dynamic model to a comprehensive 6-DOF model, including rotor flapping mechanics and 3-DOF elastic contact models for rotation and translation; this model was reduced to a simplified model for analysis. We described a PD control structure for rotation and position control, making the assumption of time-scale separation. We demonstrated that around level hover, the analytical model will be stable for a range of given flight control parameters, end-effector stiffnesses and displacements. Simulation of the full dynamic model confirms the stability transitions predicted by the analytical model.

The key insight for aerial manipulation is that for underslung end-effector contacts, high proportional attitude gain is essential for robust object interaction. Contact configurations substantially above the aircraft $\mathrm{CoG}$ produce pathological behavior, but lateral offsets do not significantly affect the stability of the system. Further work is required to validate the simulation with experiments and further the analysis for stability of coupled dynamic modes.

\section{ACKNOWLEDGMENTS}

The authors would like to thank Lael Odhner for his helpful discussion related to this work.

\section{REFERENCES}

[1] J.M. Moore, "A System for Landing an Autonomous Radio Controlled Helicopter on Sloped Terrain," masters thesis, Massachusetts Institute of Technology, 1994.

[2] L. Kaufman and E. Schultz, "The Stability and Control of Tethered Helicopters," Journal of the American Helicopter Society, Vol. 7, No. 4, 1962.

[3] G. Schmidt and R. Swik, "Automatic hover control of an unmanned tethered rotorplatform," Automatica, Vol. 10, No. 4, pp 393-394, 1974.

[4] S.-R. Oh, S. Agrawal, K. Pathak, H. Pota and M. Garrett, "Approaches for a Tether-Guided Landing of an Autonomous Helicopter," IEEE Transactions on Robotics, Vol. 22, No. 3, pp 536-544, 2006.

[5] L. Gentili, R. Naldi and L. Marconi, "Modeling and Control of VTOL UAVs Interacting With the Environment," In Proc. IEEE Conference on Decision and Control, 2008.

[6] D. Cabecinhas, R. Naldi, L. Marconi, C. Silvestre and R. Cunha, "Robust Take-Off and Landing for a Quadrotor Vehicle," IEEE/RAS International Conference on Robotics and Automation, 2010.

[7] P.E.I. Pounds and A.M. Dollar, "Hovering Stability of Helicopters With Elastic Tethers," In Proc. ASME Dynamic Systems and Control Conference, 2010.

[8] P.E.I. Pounds, D.R. Bersak and A.M. Dollar, "Grasping From the Air: Hovering Capture and Load Stability," In Proc. IEEE/RAS International Conference on Robotics and Automation, 2011.

[9] R. Mahony, T. Hamel and A. Dzul-López, "Hover Control Via Lyapunov Control for an Autonomous Model Helicopter," In Proc. Conference on Decision and Control, 1999.

[10] B. Mettler, "Identification, Modeling and Characteristics of Miniature Rotorcraft," Kluwer Academics Publisher, Nowell, MA.

[11] P.E.I. Pounds, P.I. Corke and R.E. Mahony, "Modelling and Control of a Large Quadrotor Robot," Control Engineering Practice, Vol. 18, No. 7, pp 691-699, 2010.

[12] R. Prouty, Helicopter Performance, Stability, and Control, Krieger Publishing Company, 2002.

[13] J. Lončarić, "Passive Realization of Generalized Springs," In Proc. IEEE International Symposium on Intelligent Control, 1991.

[14] F. Bullo and R.M. Murray, "Proportional Derivative (PD) Control on the Euclidean Group,” In Proc. European Control Conference, 1995. 


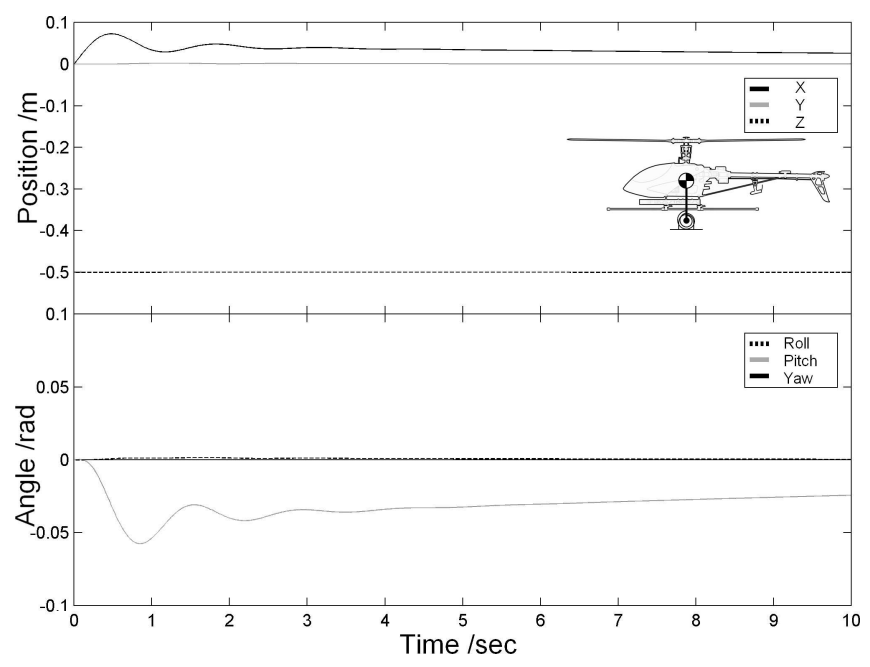

Fig. 8: Simulation $1-$ Standard gripper configuration.

$d=\left[\begin{array}{lll}0 & 0 & 0.2\end{array}\right]^{\prime} \mathrm{m}, k_{\xi}=26.6 \mathrm{Nm}^{-1}, k_{R}=2.85 \mathrm{Nmrad}^{-1}$

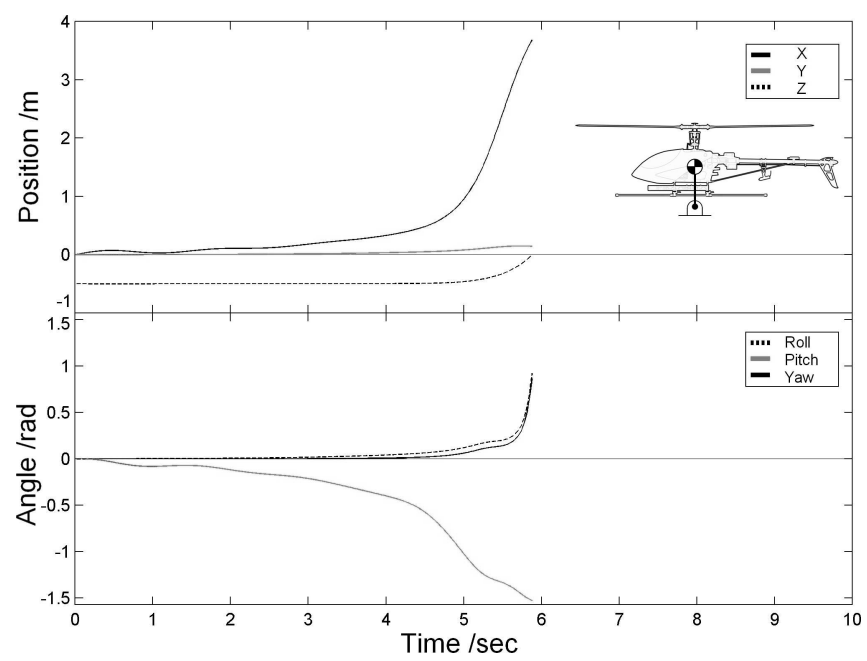

Fig. 9: Simulation $2-$ Zero rotational stiffness.

$d=\left[\begin{array}{lll}0 & 0 & 0.2\end{array}\right]^{\prime} \mathrm{m}, k_{\xi}=26.6 \mathrm{Nm}^{-1}, k_{R}=0 \mathrm{Nmrad}^{-1}$

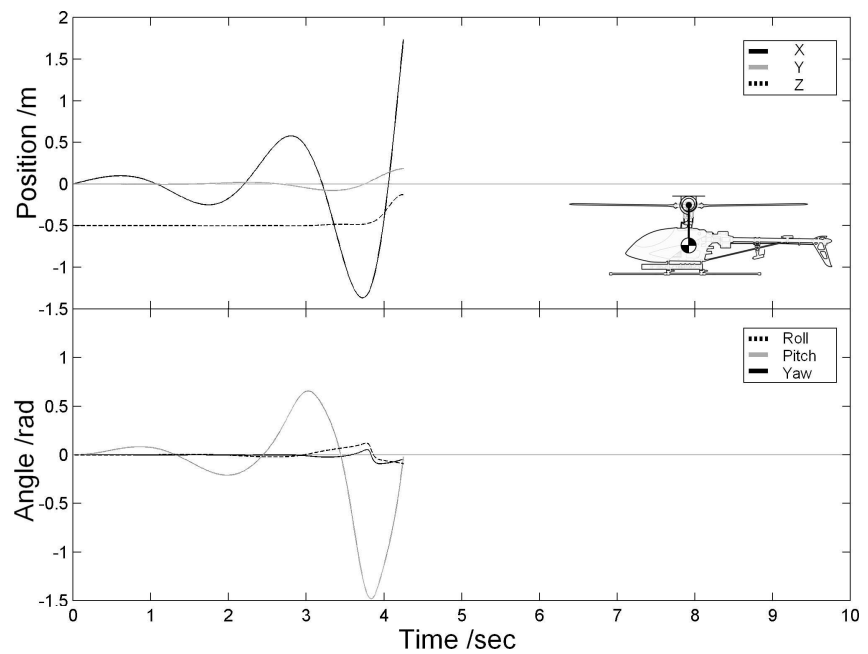

Fig. 10: Simulation 3 - Inverted contact point. $d=\left[\begin{array}{lll}0 & 0 & -0.2\end{array}\right]^{\prime} \mathrm{m}, k_{\xi}=26.6 \mathrm{Nm}^{-1}, k_{R}=2.85 \mathrm{Nmrad}^{-1}$

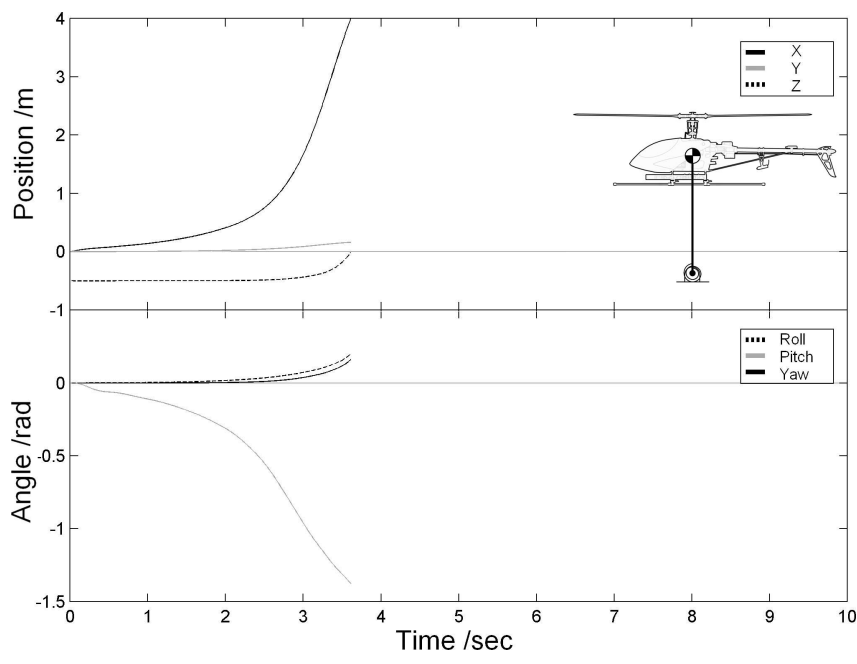

Fig. 11: Simulation $4-$ Extended vertical contact probe. $d=\left[\begin{array}{lll}0 & 0 & 1\end{array}\right]^{\prime} \mathrm{m}, k_{\xi}=26.6 \mathrm{Nm}^{-1}, k_{R}=2.85 \mathrm{Nmrad}^{-1}$

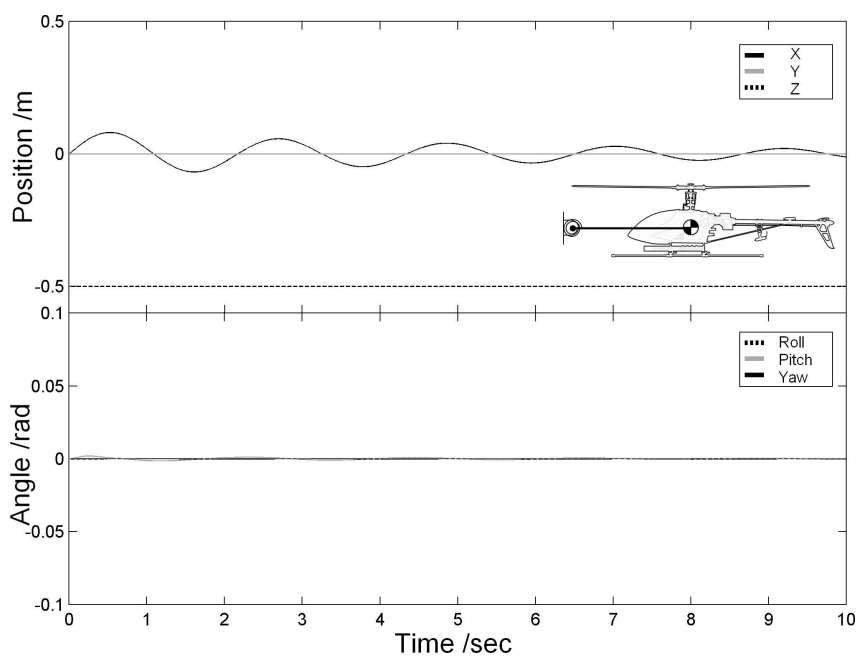

Fig. 12: Simulation 5 - Extended longitudinal contact probe. $d=\left[\begin{array}{lll}1 & 0 & 0\end{array}\right]^{\prime} \mathrm{m}, k_{\xi}=26.6 \mathrm{Nm}^{-1}, k_{R}=2.85 \mathrm{Nmrad}^{-1}$

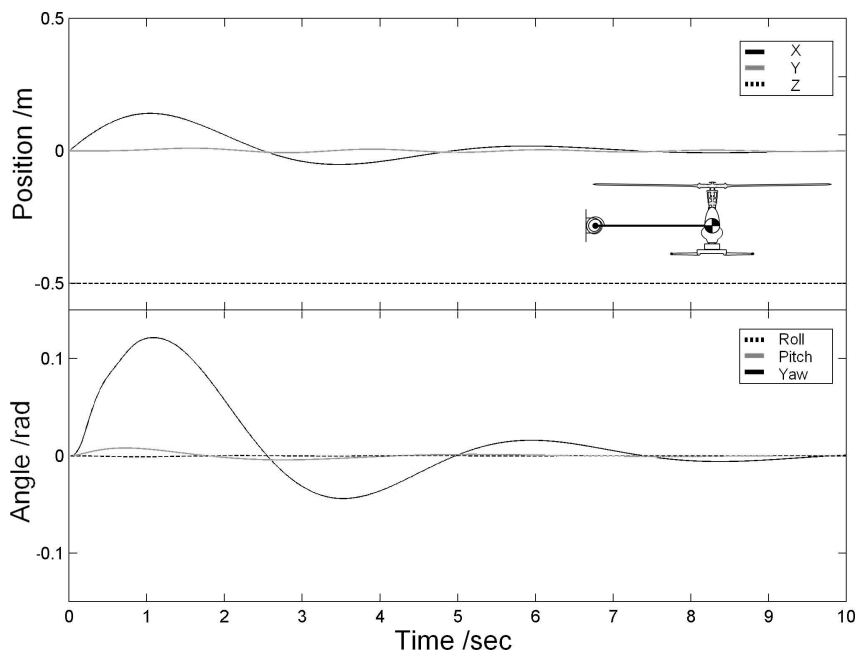

Fig. 13: Simulation $6-$ Extended lateral contact probe. $d=\left[\begin{array}{lll}0 & 1 & 0\end{array}\right]^{\prime} \mathrm{m}, k_{\xi}=26.6 \mathrm{Nm}^{-1}, k_{R}=2.85 \mathrm{Nmrad}^{-1}$ 